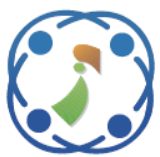

\title{
Modification of PRoPHET to Enhance Train-based Delay-Tolerant Networks Performance
}

\author{
Agus Urip Ari Wibowo, ${ }^{1,2}$ \\ Selo Sulistyo ${ }^{1 *}$ \\ I Wayan Mustika ${ }^{1}$ \\ ${ }^{1}$ Department of Electrical Engineering and Information Technology, Faculty of Engineering, \\ Universitas Gadjah Mada, Yogyakarta, Indonesia \\ ${ }^{2}$ Department of Information Technology, Politeknik Caltex Riau, Pekanbaru, Indonesia \\ * Corresponding author's Email: selo@ugm.ac.id
}

\begin{abstract}
Probabilistic Routing Protocols using History of Encounters and Transitivity (PRoPHET) assume that nodes do not move randomly but encounter destinations that have been encountered before. In some original PRoPHET and original PRoPHETv2 testing, they are superior to other routing protocols. Testing in environments that use trains as relay nodes, original PRoPHET performance is worse than the epidemic. This happens because messages delivery to other relay nodes are assumed to encounter the destination (having a larger probability of encountering destination) while the other relay nodes are moving away. The original PRoPHET and the original PRoPHETv2 performance can be improved by delivering a message to relay nodes that will encounter the destination. This can be done by delivering a message to relay nodes with lower probability values encountering the destination. The result obtained is a modified PRoPHET and a modified PRoPHETv2 performance improvement compared to the original PRoPHET and the original PRoPHETv2.
\end{abstract}

Keywords: Probabilistic routing protocol using history of encounters and transitivity (PRoPHET), PRoPHETv2, Trains, Lower probability values encountering the destination.

\section{Introduction}

The need to exchange information or messages is an unavoidable necessity on the wave of information technology. These needs include health [1], agriculture [2], and education [3]. With the Internet using Protocol/Internet Protocol Transmission Control Protocol (TCP/IP), exchanging messages using communication networks becomes easier. Network media used are cables (copper, coaxial, glass fibre) and without cable or wireless (satellite, Wireless Fidelity (Wi-Fi), etc.).

Not all places on earth have adequate infrastructure (poor infrastructure) or even no infrastructure to connect to the Internet so not everyone can exchange messages more easily. The area is generally in the countryside and in villages that are inhabited only by a small population when compared to urban areas and/or have difficult geographic conditions. Therefore, building a special network only for the area requires a relatively high cost. If the place built a network using TCP/IP then it becomes a network partition. This happens because there is no path from source to destination from/to the network to/from other networks (intermittent connectivity). The TCP/IP protocol is used with the assumption of continuous, bidirectional end-to-end path, short round-trips, symmetric data rates, and low error rates [4].

If the area lacking adequate infrastructure is served by public transportation systems, such as bus or train, then the area has opportunity to be built Wireless Local Area Networks (WLANs) network using Delay- and Disruption-Tolerant Networks (DTNs) architecture [5, 6]. DTNs architectures are developed in communication environments with the assumption of intermittent connectivity (no end-toend path between source and destination), long or 
variable delay, asymmetric data rates, and high error rates [4].

DTNs is one communication network that requires routing protocol. DTNs routing protocol pays attention to how routing is done in an efficient way in intermittently connected networks. One of the developed routing protocols is based on probabilistic, i.e. Probabilistic Routing Protocol using History of Encounters and Transitivity (PRoPHET). PRoPHET or original PRoPHET is the only DTNs routing protocol that has detailed protocol specifications that are clearly defined in IRTF Internet Design [6]. The previous encountering history with other nodes and the transitive nature of the network is used by original PRoPHET to optimize bundle delivery over the network. By A. Lindgren et al., original PRoPHET uses the assumption that nodes do not move randomly, increasing the success of message delivery with overhead of buffer usage and communication overhead at low levels [7]. The simulation results show that original PRoPHET performance is better than the epidemic.

There have been several researches that try to improve the PRoPHET performance since it was first proposed by A. Lindgren et al. S. Grasic et al. found a problem that arises in PRoPHET if a group of nodes encounter and exchange delivery predictability repeatedly that called Parking Lot Problem [8]. S. Grasic et al. recalculate the initial delivery predictability value and transitive predictability PRoPHET. The protocol is called PRoPHETv2. The simulation of PRoPHETv2 or original PRoPHETv2 used real world traffic connectivity and traces with using the Working Day Model (WDM). Sok et al. want the node with the original PRoPHET routing protocol to select and send the bundle to the neighbor node by calculating the neighbor node distance [9]. The simulation used the Random Waypoint Movement (RWP) and community models with mobility speed of 2.5-20 $\mathrm{m} / \mathrm{s}$. Lee et al. calculated the duration of encountering nodes in original PRoPHETv2 [10]. The simulation was performed at the speed of pedestrians, buses, and cars with using the WDM model. B. B. Bista and D. B. Rawat proposed an Energy Aware PRoPHET that calculates energy available on wireless devices [11]. The simulation was performed at the speed of human walking and RWP model.

A. U. A. Wibowo et al. simulated DTNs using the environment with conditions served by scheduled public transport, i.e. the train, and the train scheduled once and one-way every day [12]. The simulation showed that the original PRoPHET and the original PRoPHETv2 performance are worse than the epidemic. This is due that if the relay node encounters then the message will deliver to the relay node with a greater probability of encountering the destination. In the test, the relay node with a greater probability of encountering the destination, it is away from the destination and will encounter the same destination the next day.

In this research, the performance of the original PRoPHET and the original PRoPHETv2 was enhanced. Performance improvements were made by means of relay nodes delivering messages to other relay nodes that have a smaller probability of encountering a destination. The evaluation was done by comparing the original PRoPHET and the original PRoPHETv2 routing protocol with the modified one.

\section{Related work}

A. Lindgren et al. proposed PRoPHET which assumed that nodes do not move randomly but encounter destinations that have been encountered before based on repetitive behavior patterns [7]. This is contrast to the epidemic that considers the node to move randomly (random way-point mobility). According to the PRoPHET, when a node has visited the destination previously, it will probably visit the same destination again (community model). The area of $1500 \times 300 \mathrm{~m}$ and 50 nodes were used as a simulation to compare two routing protocols with two motion scenarios, which are random way-point mobility and community model. PRoPHET performs better than epidemic in the community model scenario. This is based on metrics of the number of messages delivered, delivery delay, and forwarded messages. PRoPHET is superior to the epidemic when it is tested with a random way-point mobility motion scenario.

S. Grasic et al. potentially improve the performance of PRoPHET by made minor modifications which resulting in what is called PRoPHETv2 [8]. In the project conducted by S. Grasic et al. in remote Swedish mountain region that implemented the DTNs system, he found that DTNs nodes in retail stores were often reconnected due to poor Wi-Fi. Each of this reconnection is counted as new encountering by each node which cause the delivery predictability for encountering nodes become too large and the mobility model have distorted patterns. This problem is solved by fixing the transitive value. The PRoPHETv2 should works in similar circumstances, therefore benchmark is required. The NC4 project was then used as the benchmark. The order of delivery rate from the 
largest to the smallest is PRoPHETv2, epidemic, and PRoPHET based on the test using Opportunistic Network Environment (ONE) simulator [13] and a Working Day Model (WDM). Meanwhile, the order from the smallest to the largest overhead ratio is PRoPHETv2, PRoPHET, and epidemic. Additionally, small changes will show that PRoPHETv2 is better than PRoPHET.

Sok et al. want the node with the original PRoPHET routing protocol to select and send the bundle to the neighbor node by calculating the neighbor node distance [9]. If two or more nodes encounter and have the same delivery predictability but the distance of the nodes to different source nodes will cause a low delivery ratio, high delay, and high overhead. Sok et al. offers Distance-based PRoPHET (DiPRoPHET) which modifies original PRoPHET by adding the application of cross-layer DTNs to take distance values. DiPRoPHET simulated with random and community model and speed of $2.5-20 \mathrm{~m} / \mathrm{s}$ through an NS2 simulation. The result is that the delivery ratio increases and the delay and overhead bundle decrease.

Lee et al. calculated the duration of encountering nodes in original PRoPHETv2 [10]. Lee et al. added calculation of encountering duration and the duration needed to send a message on delivery predictability. The simulation used the ONE simulator that compared the proposed PRoPHETv2 with original PRoPHETv2. The metrics produced are delivery ratio and overhead ratio. The motion model used is the WDM at the speed of pedestrians, buses and cars. Proposed PRoPHETv2 by Lee et al. produce a better delivery probability and overhead ratio than original PRoPHETv2. Delivery probability of proposed PRoPHETv2 is greater than original PRoPHETv2 and overhead ratio of proposed PRoPHETv2 is smaller than original PRoPHETv2. The greater the bandwidth, the greater the delivery probability and overhead ratio. The greater the average message size, the smaller delivery probability and overhead ratio.

B. B. Bista and D. B. Rawat proposed PRoPHET by considering to the energy available on wireless devices [11]. The main issue is the use of wireless devices that are ubiquitously. This is a concern of the research because nodes that die earlier will reduce the possibility of sending messages on the network. B. B. Bista and D. B. Rawat not only concern to the energy available but also the available empty buffers. B. B. Bista and D. B. Rawat propose the name of the routing protocol as Energy Aware PRoPHET (EA-PRoPHET). The simulation was performed using ONE with human walking speed and the RWP model. The results obtained are increased message delivery in the network because the device uses less energy so the network age increases. According to B. B. Bista and D. B. Rawat that the most important thing on mobile devices that use batteries is the energy and empty buffers available.

\section{PRoPHET}

Node historical concepts and transitivity properties are used in this routing protocol with $P_{(A, B)}$ as the probability that two nodes of A and B are encountering each other. This probability, called the delivery predictability, is shown in Eq. (1) and $P_{\text {init }}$ is given a constant initial value [7].

$$
\begin{aligned}
& P_{(A, B)}=P_{(A, B) \text { old }}+\left(1-P_{(A, B) \text { old }}\right) \times P_{\text {init }}, \\
& P_{\text {init }} \in[0,1]
\end{aligned}
$$

The $P_{(A, B)}$ is the current delivery predictability node while $P_{(A, B) \text { old }}$ is the previous delivery predictability node. The value of $P_{(A, B)}$ between 0 and $1, P_{(A, B)} \in[0,1]$. However, it is updated at successive encountering between the same nodes and can decrease over time when nodes do not encounter. Eq. (2) shows a predictable value decreasing, where $\gamma$ is the aging constant and $k$ is the time elapsed since the last aging. This causes the value of $P_{(A, B)}$ to be heuristic [14].

$$
P_{(A, B)}=P_{(A, B) o l d} \times \gamma^{k}, \gamma \in[0,1)
$$

The node A often encounters node B and node B often encounters node $\mathrm{C}$, thus transitivity property of $P_{(A, C)}$ of the delivery predictability is made. The Eq. (3) below shows the transitivity where $\beta$ is a scaling constant that determine the impact of transitivity on delivery predictability.

$$
\begin{aligned}
& P_{(A, C)}=P_{(A, C) \text { old }}+\left(1-P_{(A, C) \text { old }}\right) \times P_{(A, B)} \times \\
& P_{(B, C)} \times \beta, \beta \in[0,1]
\end{aligned}
$$

Delivery predictability values are calculated on all network nodes and the value is updated each time the node encounters. This is used to decide whether it messages are delivered or not during communication. Delivery predictability information stored between two nodes will be exchange every time both nodes encounter. This delivery predictability information is then used by both nodes to update their delivery predictability prediction information. Therefore, the message is delivered to another node when the message destination delivery 
predictability is higher on other node. The proposed values are $P_{\text {init }}=0.75, \beta=0.25$, and $\gamma=0.98$ [7].

There is a Parking Lot Problem on PRoPHET based on research by S. Grasic et al. [8]. The observation on this problem shows that the occurring movements do not fit typical human movement patterns for hours or days. The changing Wi-Fi signal is causing re-connection of devices in the parking lot that is considered as new encountering. S. Grasic et al. examined that if $\beta>0$, delivery predictability values in Eq. (3) would always be enlarged.

This problem is solved by protocol improvement version in PRoPHETv2 with $P_{\text {init }}$ calculated as follows.

$$
P_{\text {init }}= \begin{cases}P_{\max } \times \Delta_{j} / \Delta, & 0 \leq \Delta_{j} \leq \Delta \\ P_{\max }, & \Delta<\Delta_{j}\end{cases}
$$

$\Delta_{\mathrm{j}}$ is the time since the last encounter with node $\mathrm{j}$ and $\Delta$ is the average time interval between two consecutive connections for a given network scenario. The predicted transitive evaluation is changed to:

$$
P_{(A, C)}=\max \left\{P_{(A, C) \text { old }}, P_{(A, B)} \times P_{(B, C)} \times \beta\right\}
$$

Eq. (5) stabilizes the enlarged delivery predictability value unexpectedly.

\section{Proposed improvement}

Based on [12] it appears that the original PRoPHET and the original PRoPHETv2 are worse than the epidemic. This is because if the relay nodes encounter then the relay nodes with smaller delivery predictability will deliver messages to relay nodes that have larger delivery predictability.

Let TR 9 (Table 1 [15]) be the relay node of station A (Fig. 1) and TR 20 (Table 1) as the relay nodes of station B (Fig. 1). In the railway, there is a village close to the station A. TR 9 and TR 20 carrying messages for the village. TR 9 passes the village earlier than TR 20. Short contact time between train 9 and village causes not all messages can be delivered from TR 9 to the village. As a result of the encountering, the value of delivery predictability TR 9 with the village is increased (Eq. (1)). At the same time the value of delivery predictability TR 20 with the village is reduced (Eq. (2)).

At a place, there was an encountering between TR 9 and TR 20. The message for the village from TR 20 was duplicated and delivered to TR 9 because

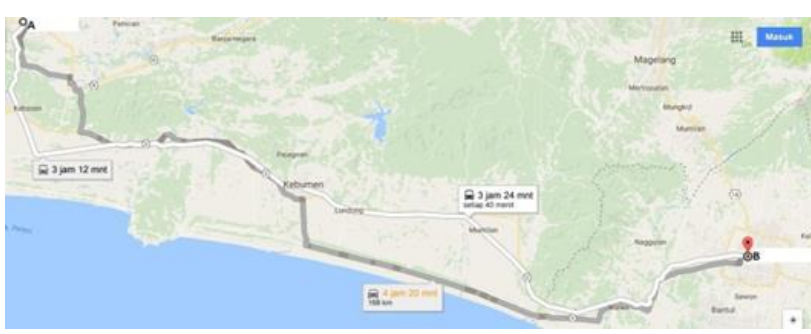

Figure.1 The railway station A-station B of Google map

Table 1. Train schedule A-B

\begin{tabular}{|l|l|l|}
\hline Time & Route & Name \\
\hline 00:26 & A-B & TR 1 \\
\hline 01:15 & A-B & TR 2 \\
\hline 01:55 & A-B & TR 3 \\
\hline $02: 57$ & A-B & TR 4 \\
\hline 03:20 & A-B & TR 5 \\
\hline 07:00 & B-A & TR 11 \\
\hline $08: 00$ & B-A & TR 12 \\
\hline $08: 57$ & B-A & TR 13 \\
\hline 09:00 & B-A & TR 14 \\
\hline 11:42 & A-B & TR 6 \\
\hline
\end{tabular}$\quad$\begin{tabular}{ll|l|l|}
\hline 14:00 & A-B & AR 7 \\
\hline $17: 45$ & B-A & TR 8 \\
\hline $18: 35$ & B-A & TR 16 \\
\hline 20:00 & B-A & TR 17 \\
\hline $20: 35$ & B-A & TR 18 \\
\hline $20: 57$ & B-A & TR 19 \\
\hline $21: 40$ & A-B & TR 9 \\
\hline $22: 00$ & B-A & TR 20 \\
\hline $22: 37$ & A-B & TR 10 \\
\hline
\end{tabular}

delivery predictability TR 9 with the village is bigger than the delivery predictability TR 20 with the village. Messages for the village of TR 9 that have not been delivered are not duplicated. This causes messages for the village from TR 9 that have not been delivered will never be delivered until the messages are discarded because the message's live time has passed the specified time for the buffer not to be full.

Based on the above problem then the original PRoPHET and the original PRoPHETv2 need to be modified so that the message is duplicated and delivered to the relay node with a smaller delivery predictability. The results of this change produce the modified PRoPHET and the modified PRoPHETv2. The original PRoPHET and the original PRoPHETv2 use the principle that the message is delivered to another relay node if the message destination delivery predictability is higher in the other relay node. The modified PRoPHET and modified PRoPHETv2 use the principle of the message delivered to other relay nodes if the message destination delivery predictability is smaller in the other relay nodes.

\section{Performance metric}

K. Fall states that DTNs as message-oriented reliable overlay architecture located above the transport layer on the connected network [16]. If on a TCP/IP network using the term packet then DTN uses a message [16].

DTNs have the following characteristics [17]: 
1. The absence of an end-to-end communication path then delivering messages using hop-by-hop

2. Because it can carry messages in a long time and delivered to other nodes in case of contacts it requires the ability to store messages

3. Message replication not forwarding

4. Messages can experience a high delivery latency that is usually several hours

Table 2. Parameter equations

\begin{tabular}{|c|c|}
\hline Parameter & Definition \\
\hline$M$ & $\begin{array}{l}\text { a set of messages created by the } \\
\text { source node in the network }\end{array}$ \\
\hline$M_{d}$ & $\begin{array}{l}\text { the set of messages successfully } \\
\text { received by the destination node in } \\
\text { the network where } M_{d} \subseteq M\end{array}$ \\
\hline$t_{i}$ & $\begin{array}{l}\text { the time the message was created by } \\
\text { the source node }\end{array}$ \\
\hline$t_{i}^{\prime}$ & $\begin{array}{l}\text { the time the message was received } \\
\text { successfully by the destination node }\end{array}$ \\
\hline $\begin{array}{c}\text { delivery } \\
\text { probability }(d p)\end{array}$ & $\begin{array}{l}\text { the comparison of the number of } \\
\text { messages with the size of } i \\
\text { successfully received by the } \\
\text { destination node compared to the } \\
\text { number of message sizes } i \text { created } \\
\text { by the source node }\end{array}$ \\
\hline $\begin{array}{l}\text { latency average } \\
(l a)\end{array}$ & $\begin{array}{l}\text { the average time required by } \\
\text { messages of size } i \text { ranges from being } \\
\text { created to successfully received by } \\
\text { the destination node }\end{array}$ \\
\hline $\begin{array}{l}\text { overhead ratio } \\
\text { (or) }\end{array}$ & $\begin{array}{l}\text { the number of copies of messages } \\
\text { delivered to the destination node are } \\
\text { compared to messages successfully } \\
\text { received by the destination node }\end{array}$ \\
\hline $\begin{array}{l}\text { average of } \\
\text { delivery } \\
\text { probability } \\
\left(d p_{\text {avg }}\right)\end{array}$ & $\begin{array}{c}\text { a comparison of the number of all } \\
\text { message sizes successfully received } \\
\text { by the destination node compared to } \\
\text { the number of all message sizes } \\
\text { successfully created by the source } \\
\text { node }\end{array}$ \\
\hline $\begin{array}{c}\text { average of } \\
\text { latency average } \\
\quad\left(l_{\text {avg }}\right)\end{array}$ & $\begin{array}{c}\text { the average time required by all } \\
\text { message sizes starting from created } \\
\text { until received by the destination } \\
\text { node }\end{array}$ \\
\hline $\begin{array}{l}\text { average of } \\
\text { overhead ratio } \\
\quad\left(\text { or }_{\text {avg }}\right)\end{array}$ & $\begin{array}{l}\text { a comparison of the number of } \\
\text { copies of all message sizes delivered } \\
\text { to the destination node compared to } \\
\text { all messages successfully received } \\
\text { by the destination node }\end{array}$ \\
\hline$r_{i}$ & $\begin{array}{c}\text { number of copies of messages in the } \\
\text { network where } 0 \leq r_{i} \leq N\end{array}$ \\
\hline$N$ & $\begin{array}{l}\text { the total number of nodes in the } \\
\text { network }\end{array}$ \\
\hline$n$ & $\begin{array}{c}\text { the number of message sizes is } 19 \text { ( } 1 \\
\text { Kilo Byte, } 2 \text { Kilo Bytes, up to } 100 \\
\text { Kilo Bytes) }\end{array}$ \\
\hline
\end{tabular}

Routing protocols DTNs are evaluated and analyzed using the following performance measures [14]:

1. Delivery Probability $(d p)$

$d p=\frac{M_{d}}{M}$

2. Latency Average (la)

$l a=\frac{\sum_{i=1}^{M_{d}}\left(t_{i}^{\prime}-t_{i}\right)}{M_{d}}$

3. Overhead Ratio (or)

or $=\frac{\sum_{i=1}^{M} r_{i}-M_{d}}{M_{d}}$

4. Average of Delivery Probability ( $\left.d p_{\text {avg }}\right)$

$d p_{a v g}=\frac{\sum_{j=1}^{n} M_{d}}{\sum_{j=1}^{n} M}$

5. Average of Latency Average $\left(l a_{a v g}\right)$

$l a_{\text {avg }}=\frac{\sum_{j=1}^{n} \sum_{i=1}^{M_{d}}\left(t_{i}^{\prime}-t_{i}\right)}{\sum_{j=1}^{n} M_{d}}$

6. Average of Overhead Ratio (or avg)

$o r_{a v g}=\frac{\sum_{j=1}^{n}\left(\sum_{i=1}^{M} r_{i}-M_{d}\right)}{\sum_{j=1}^{n} M_{d}}$

\section{Simulation setup}

The simulation uses ONE [13] and requires scenarios. The scenario is used as in [12]. Scenarios created using local maps with a real environment (Fig. 1). In this research using the railway line station A-station B with a distance of 166 Kilometres. Ten trains depart from station A to station B and ten from station B to station A. Each train has a designated departure schedule and in one day only moves one direction only, does not return to the place of departure (Table 1). The train from station A stops in station B and the train from station B stops in station A. The difference with [12] is that the train speed is raised to $83 \mathrm{Km} /$ hour or 2.306 $\mathrm{m} / \mathrm{second}$ for the ONE scale. The size and number of messages using parameter [12]. The ONE parameters are transmitted range, routing protocols, 
and the buffer size is 50 meters, epidemic, original PRoPHET, modified PRoPHET, original PRoPHETv2 and modified PRoPHETv2, and 10 Mega Bytes respectively. Other ONE parameters are same as [12].

The original PRoPHET and original PRoPHETv2 use the principle that the message is delivered to another node if the message destination delivery predictability is higher in that other node. The modified PRoPHET and the modified PRoPHETv2 use the principle of the message to be delivered to another node if the message destination delivery predictability is smaller on that other node.

\section{Result}

The simulation results are graphic delivery probability (Fig. 2), latency average (Fig. 3), overhead ratio (Fig. 4), average value of delivery probability (Fig. 5), average value of latency average (Fig. 6), and average value of overhead ratio (Fig. 7). Fig. 2-4 contains two types of graphs, namely graph (a) for the dissemination of messages from station to village and graph (b) gathering of messages from village to station.

Fig. 2 shows that the delivery probability of the modified PRoPHET and the modified PRoPHETv2 are better than original. In Fig. 2(a) graph of modified PRoPHET and modified PRoPHETv2 approaching epidemic but still smaller. The original PRoPHET failed to deliver messages from station to village. In Fig. 2(b) the modified PRoPHETv2 graph almost coincides with the epidemic. Although the modified PRoPHET graph is lower than the modified PRoPHETv2 and epidemic but is almost twice as large as than the original PRoPHET and the original PRoPHETv2.

In the latency average graph (Fig. 3) it appears that to obtain a better delivery probability value than the original PRoPHET and the original PRoPHETv2 then the modified PRoPHET and the modified PRoPHETv2 require a longer time to deliver the message. The latency average value of modified PRoPHETv2 almost coincides with the epidemic in Fig. 3 (a). In Fig. 3 (b) the latency average value of modified PRoPHETv2 is lower than epidemic at message sizes 1 Kilo Byte to 3 Kilo Bytes and higher than the epidemic in sizes 4 Kilo Bytes to 100 Kilo Bytes. Although the modified PRoPHET gets the delivery probability better than the original PRoPHET but requires a lower latency average than the original PRoPHET.

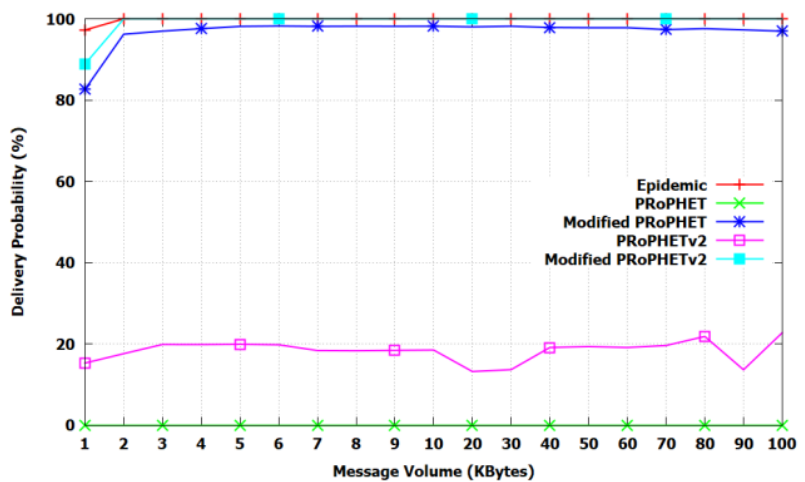

(a)

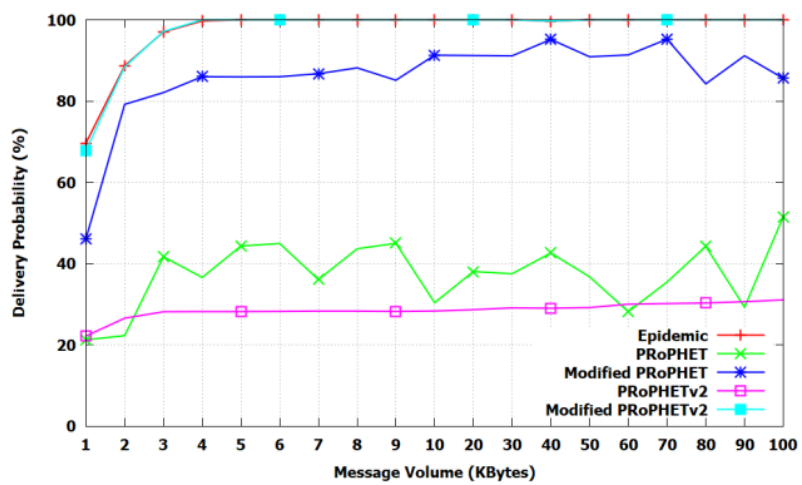

(b)

Figure. 2 Delivery probability: (a) dissemination of messages from station to village and (b) gathering of messages from village to station

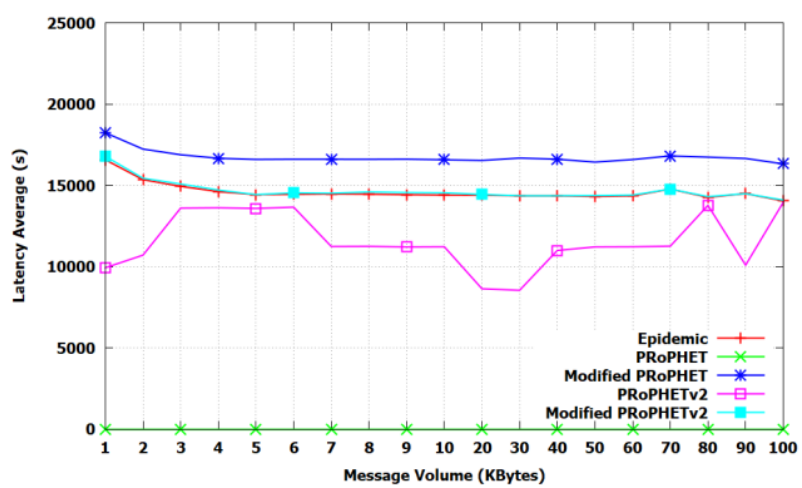

(a)

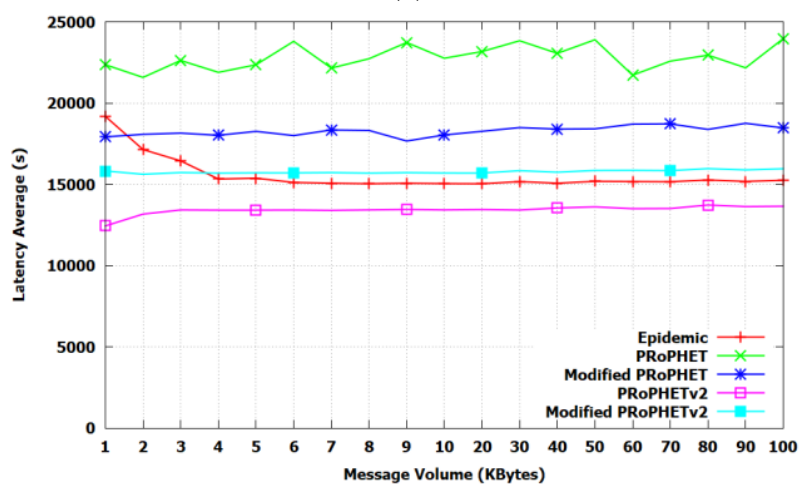

(b)

Figure.3 Latency average: (a) dissemination of message from station to village and (b) gathering of messages from village to station 
In the overhead ratio graph (Fig. 4) it appears that to obtain better delivery probability values, the modified PRoPHET and the modified PRoPHETv2 require a smaller overhead ratio than the epidemic, the original PRoPHET, and the original PRoPHETv2. This proves that replication in the modified PRoPHET and the modified PRoPHETv2 are better than the epidemic, the original PRoPHET, and the original PRoPHETv2.

The average value for all message sizes is shown in Fig. 5, Fig. 6, and Fig. 7. In Fig. 5 the modified PRoPHET and the modified PRoPHETv2 obtained an average delivery probability value is smaller than the epidemic. In Fig. 7 the modified PRoPHET and the modified PRoPHETv2 obtained an average overhead ratio smaller than the epidemic. This indicates that the modified PRoPHET and the modified PRoPHETv2 require a smaller buffer than is required by the epidemic. The modified PRoPHET and the modified PRoPHETv2 obtained higher average probability delivery and smaller overhead ratios than the original PRoPHET and the original PRoPHETv2.

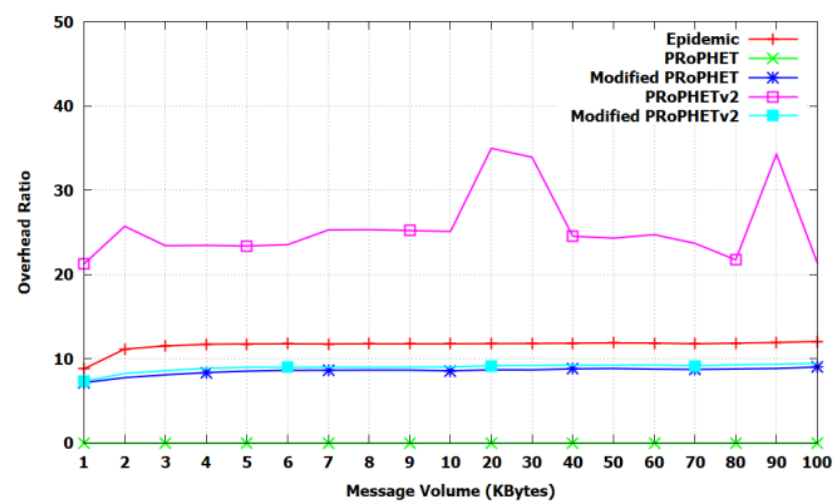

(a)

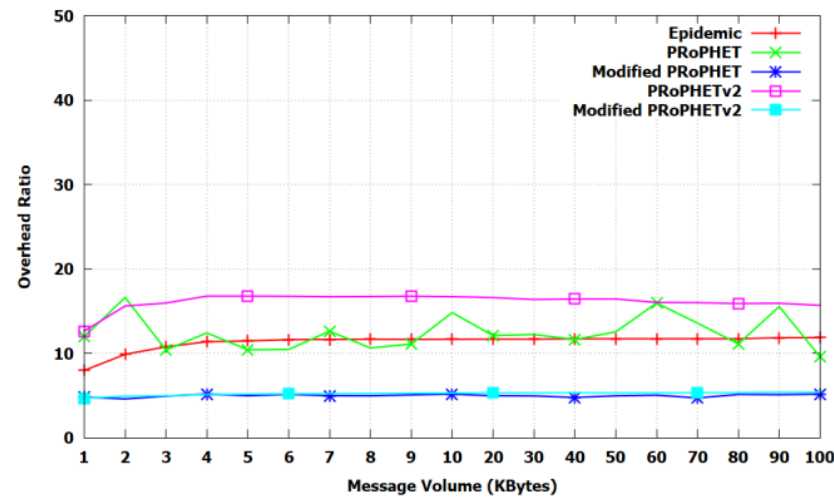

(b)

Figure.4 Overhead ratio: (a) dissemination of message from station to village and (b) gathering of messages from village to station

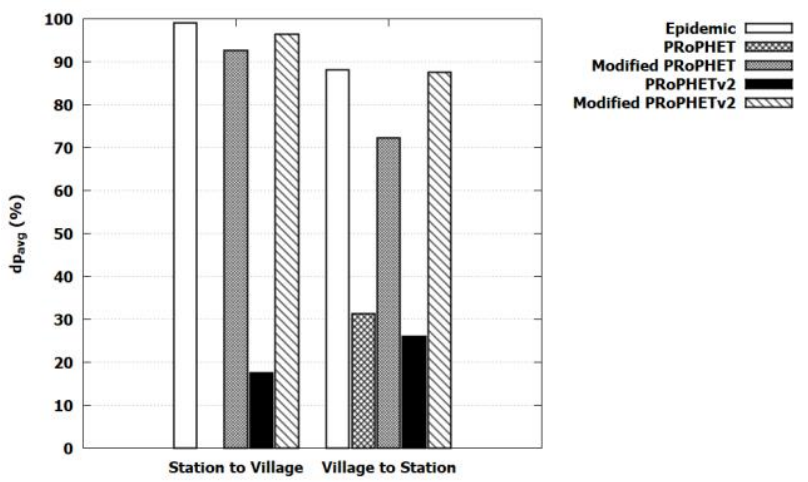

Figure.5 Average value of delivery probability for all message sizes

\section{Conclusions}

In this research using local map with a realistic environment and using scheduled trains [12]. Trains move in one direction and once a day as relay nodes to disseminate messages from station to village and gather messages from village to station. The routing protocol used is the original PRoPHET and the original PRoPHETv2. Delivery probability, latency average, and overhead ratio metrics are worse than an epidemic.

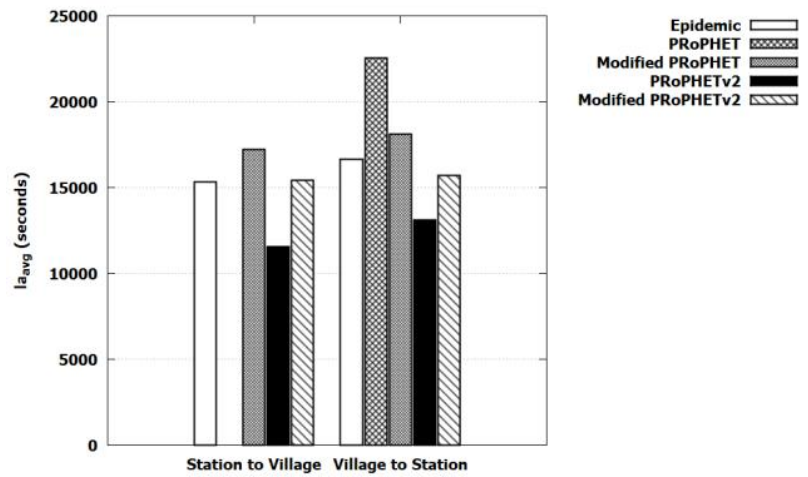

Figure.6 Average value of latency average for all message sizes

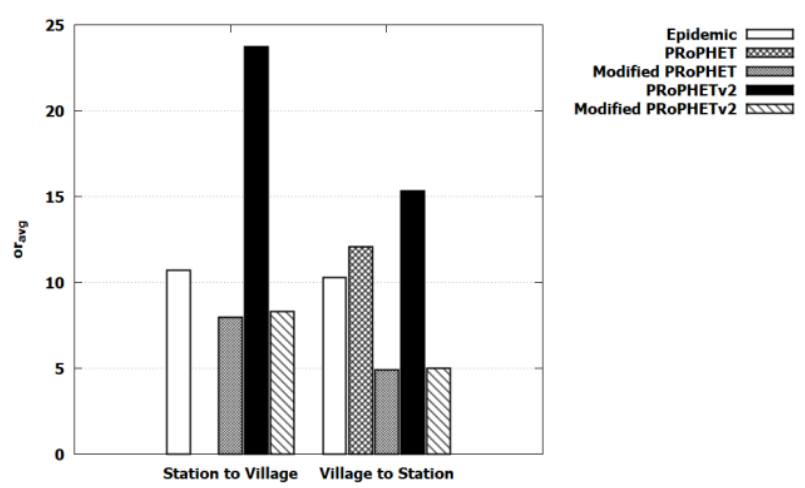

Figure.7 Average value of overhead ratio for all message sizes 
Relay nodes that use the original PRoPHET and the original PRoPHETv2 deliver messages to other relay nodes with a higher delivery predictability. The modified PRoPHET and the modified PRoPHETv2 make relay nodes deliver messages to other relay nodes with a lower delivery predictability. The modified PRoPHET and the modified PRoPHETv2 successfully outperform the original PRoPHET and the original PRoPHETv2 on three performance metrics unless the original PRoPHETv2 is better in the latency average metric. The modified PRoPHET and the modified PRoPHETv2 are more suitable for use in environments that use scheduled trains that move in one direction and once a day as node relays than the original PRoPHET and the original PRoPHETv2.

The delivery probability metric value of the modified PRoPHET and the modified PRoPHETv2 successfully approach the epidemic value. Epidemic outperforms at the latency average metric value but the modified PRoPHET and modified PRoPHETv2 outperform at the overhead ratio metric value.

The future research is to test the modified PRoPHET and the modified PRoPHETv2 in a realistic environment using buses or cars as a relay node.

\section{Acknowledgments}

This work was partially supported by Ministry of Research, Technology, and Higher Education, Indonesia under the PUPT grant number 751/UN1P.III/LT/DIT-LIT/2016 and was conducted in the Department of Electrical Engineering and Information Technology, Faculty of Engineering, Universitas Gadjah Mada, Yogyakarta, Indonesia.

\section{References}

[1] S. Syed-Abdul, J. Scholl, P. Lee, W-S Jian, DM Liou, and Y-C Li, "Study on the potential for delay tolerant networks by health workers in low resource settings", Computer Methods and Programs in Biomedicine, Vol.107, No.3, pp.557-564, 2012.

[2] U. R. Ahuja, R. Jain, S. Chauhan, Amarjit, P. Narayan, and K. R. Chaudhry, "SocioEconomic Impact of Mobile Phone in Agriculture: A Case Study of Karnal District", In: Proc. of 2015 2nd International Conference on Computing for Sustainable Global Development, pp.1176-1179, 2015.

[3] S. Chaklader, J. Alam, M. Islam, and A. S. Sabbir, "Bridging Digital Divide:Village wireless LAN, a low cost network infrastructure solution for digital communication, information dissemination \& education in rural Bangladesh", In: Proc. of 2013 International Conference on Advances in Electrical Engineering (ICAEE), pp.277-281, 2013.

[4] F. Warthman and W. Associates, "Delay-and Disruption-Tolerant Networks (DTNs): A Tutorial", Interplanetary Internet Special Interest Group, Vol.2, pp.1-33, 2012.

[5] V. Cerf, S. Burleigh, A. Hooke, L. Torgerson, R. Durst, K. Scott, K. Fall, and H. Weiss, "Delay-tolerant networking architecture", $R F C$ 4838, 2007.

[6] A. Lindgren, A. Doria, E. Davies, and S. Grasic, "Probabilistic routing protocol for intermittently connected networks", Internet Draft draft-lindgren-dtnrg-prophet-09.txt, 2011.

[7] A. Lindgren, A. Doria, and O. Schelen, "Probabilistic routing in intermittently connected networks", In: Proc. of ACM SIGMOBILE Mobile Computing and Communications Review, Vol.7, No.3, pp.1920, 2003.

[8] S. Grasic, E. Davies, A. Lindgren, and A. Doria, "The Evolution of a DTN Routing Protocol PRoPHETv2", In: Proc. of the 6th ACM workshop on Challenged Networks, pp.27-30, 2011.

[9] P. Sok, S. Tan, and K. Kim, "PRoPHET Routing Protocol Based on Neighbor Node Distance Using a Community Mobility Model in Delay Tolerant Networks", In: Proc. of 2013 IEEE $10^{\text {th }}$ International Conference on High Performance Computing and Communications \& 2013 IEEE International Conference on Embedded and Ubiquitous Computing, pp.1233-1240, 2013.

[10] H.-J. Lee, J.-C. Nam, W.-K. Seo, Y.-Z. Cho, and S.-H. Lee, "Enhanced PRoPHET routing protocol that considers contact duration in DTNs", In: Proc. of 2015 International Conference on Information Networking, pp.523-524, 2015.

[11] A. B. Bista and D. B. Rawat, "EA-PRoPHET: An Energy Aware PRoPHET-Based Routing Protocol for Delay Tolerant Networks", In: Proc. of 2017 IEEE 31st International Conference on Advanced Information Networking and Applications, pp.670-677, 2017.

[12] A. U. A. Wibowo, S. Sulistyo, I W. Mustika, and A. Hafid, "Performance Evaluation of DTNs Routing Protocols on Train System by Variations in Message Volume", International 
Journal of Intelligent Engineering and Systems, Vol.10, No.3, pp.200-209, 2017.

[13] A. Keränen and J. Ott, "Increasing reality for DTN protocol simulations", Technical Report, Helsinki University of Technology, Networking Laboratory, 2007.

[14] S. Misra, B. K. Saha, and S. Pal, "Delay Tolerant Routing and Applications", in Opportunistic Mobile Networks, Cham: Springer International Publishing, pp.23-52, 2016.

[15] P. T. K. A. I. (Persero), Jadwal Perjalanan Kereta Api 2015, Jl. Perintis Kemerdekaan No. 1 Bandung: PT. Kereta Api Indonesia, 2015.

[16] K. Fall, "A delay-tolerant network architecture for challenged internets", In: Proc. of the 2003 conference on Applications, technologies, architectures, and protocols for computer communications, Intel Research Berkley: ACM, pp.27-34, 2003.

[17] S. Misra, B. K. Saha, and S. Pal, "Origins and Characteristics", in Opportunistic Mobile Networks, Cham: Springer International Publishing, pp.3-21, 2016. 